\title{
Decentralized Mobile Sensor Navigation for Hole Healing Policy in Wireless Hybrid Sensor Networks
}

\author{
Fu-Tian Lin ${ }^{1,2}$, Chu-Sing Yang ${ }^{1}$, Tien-Wen Sung ${ }^{1}$ and Bi-jar Lin ${ }^{2}$ \\ ${ }^{1}$ Institute of Computer and Communication Engineering, Department of Electrical \\ Engineering, National Cheng Kung University, Taiwan (R.O.C.) \\ ${ }^{2}$ Tung Fang Design University, Taiwan, (R. O. C.) \\ q3897112@mail.ncku.edu.tw, csyang@ee.ncku.edu.tw, q38994025@mail.ncku.edu.tw \\ and rlattaiwan@gmail.com
}

\begin{abstract}
This paper proposes a method to deal with holes healing problems in wireless hybrid sensor networks. With recent advances in mobile platforms, hybrid sensor networks including static and mobile sensors are becoming very popular technology. In this paper, we investigate the coverage hole healing efficiency and propose a method to improve the efficiency. Specifically, mobile sensors can dynamically move to the un-coverage areas, improving both the coverage rate and the quality of service in WSN applications. During the process of mobile sensors moving, obstacles may exist in the environment. In order to reach the anticipated destination effectively, mobile sensors need to find an obstacle-free path for movement. To do this, we proposed an obstacle-free and geographic-free decentralized dispatch scheme $(O G D D)$ to select and navigate the mobile sensors to participate the healing work. Experimental results reveal that the proposed scheme can be efficiently executed.
\end{abstract}

Keywords: Hybrid sensor network, holes healing, sensors navigation, obstacle-free, geographic-free

\section{Introduction}

Wireless sensor networks (WSNs) are a very popular technology for monitoring in risky areas. Several researchers have investigated the deployment of the sensor nodes, which is one of the most important issues in WSNs applications. Most related works regard the deployment problem as a coverage problem. Sensor coverage is typically defined according to the sensing area [1] [1]. It is fundamentally inter-related in a sensor network. Numerous deployment algorithms have been proposed to increase the coverage rate in the sensor field $[3,5]$. Since sensors typically operate on batteries and are thus limited in their active lifetime, the energy problem causes the coverage holes appearance. With recent advances in mobile platforms, hybrid sensor networks which comprise static and mobile sensors are becoming increasingly attractive. Mobile sensors are advantageous because they can move to cover the uncovered area to increase the coverage. Chang et al., [5] design a deployment scheme, SelfDeployment by Density Control (SDDC), which utilizes the mobile sensors to balance the sensors density, and can prolong the network lifetime. Wang et al., [6] discuss two related deployment problems, which consist of sensor placement and sensor dispatch in hybrid sensor networks to increment both the coverage rate and the network lifetime. However, wireless sensors usually have resource constraints, like powered battery, computing capability and communication range. Most optimal sensor placement schemes need more computing 
capability of sensors and the geographic information of obstacle. Nevertheless, in most of WSNs applications, sensor nodes are randomly deployed in the target area without any positioning device, since GPS and other localization devices require relatively high-energy budget for the sensor nodes. Hence we should find an obstacle-free moving path with low computational complexity for mobile sensor navigation, and considering a distribution dispatch method to reduce the local energy excessive consumption. This work is a decentralized, obstacle-free and graphic-free dispatch scheme with shortest path routing for mobile sensors.

The remainder of this paper is organized as follows. Section 2 introduces the preliminary of network. Section 3 elucidates the proposed navigation scheme and the mobile sensors selection policy. Section 4 summarizes the simulation results. Section 5 draws conclusions.

\section{Preliminary}

This section introduces the mobile sensor dispatch problem in hybrid sensor networks for holes healing. The main advantage is using the mobile sensors can move to monitor uncovered areas to increase the coverage, the uncovered areas called a hole alternately. In fact, the moving path of mobile sensors in field with obstructions is an important issue. In this work scenario, the static sensors are installed in a monitoring area in advance of monitoring, a few number of mobile sensors distributed over the monitoring field, all buildings, trees, and houses regarded as obstacles, and the sensors can detect the un-coverage area by using the hole detecting algorithm.

Due to the fact that the sensors usually have resource constraints, an important problem is how to find a moving path with minimum energy consumption and no interference by the obstacles. Figure 1 shows mobile sensor movement hindered by an obstacle problem. A mobile moves to the predetermine target. Generally, it uses a directly direction movement. However, an obstacle is available for the mobile sensor moving path. Thus, if no pre-route navigation path for the mobile sensor, it will be interfered by the obstacle and the mobile sensor cannot move to the destination.

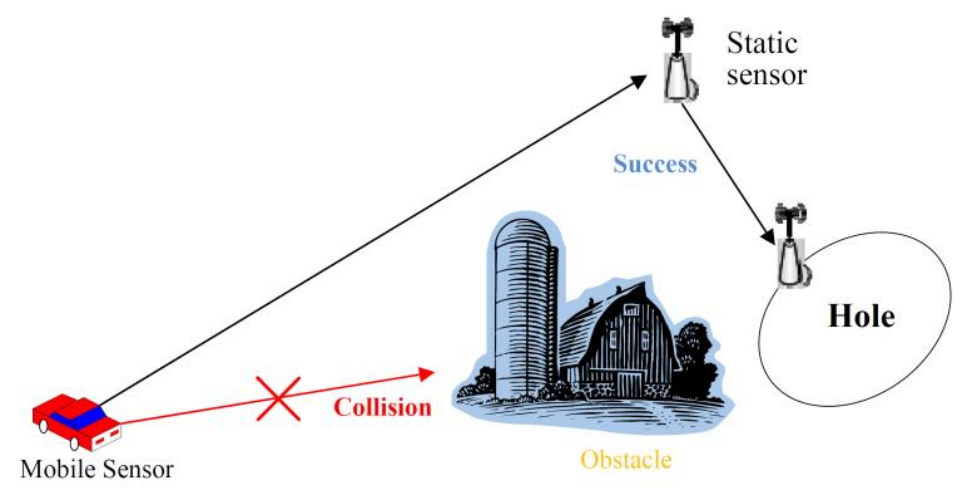

Figure 1. Illustrate the Obstacle Problem in Direct Moving Path

\section{Mobile Sensors Selection Policy and Navigation Scheme}

The monitoring area is modeled in 2D Coordinate, and obstacles may exist inside with arbitrary sharp and obstacles, but do not separate the monitoring area. In this work, both static and mobile sensors have resource limitations and no geographic information about the obstacles. If a hole is detected, then hole healing task will be performed. During the heal 
processing, mobile sensors have to move to the hole. Thus, it needs to find a path for the mobile sensors to move. Despite lack of the geographic information, it has to avoid any obstacles.

Since several articles discuss holes detection schemes [7]-[9], we applied one of them in this work. Based on the holes detection, $S$ denotes a group set, which consist of the boundary nodes of the hole. In $S$, one of the boundary nodes has maximum residual energy selected as header. An example is depicted in Figure 2, which illustrates the members of $S, 15,17,20,21$ and 16, as well as the boundary node 15 , the header of $S$. Specifically, the header has to evaluate the size of the hole and calculate how many mobile nodes needed for hole healing task. Here, for one hole, we defined $S^{\prime}$ as the subset of $S\left(S^{\prime} \subseteq S\right)$, which consisted the participation boundary nodes for healing task. When mobile nodes arrive at the participation boundary node, they enter into the hole, an attractive/repulsive method to be used in the hole healing which is proposed in the article [10][11]. This task assumes that the selected boundary nodes are the destinations of the mobile sensors, because those boundary nodes are close to coverage hole and easy to enter to it.

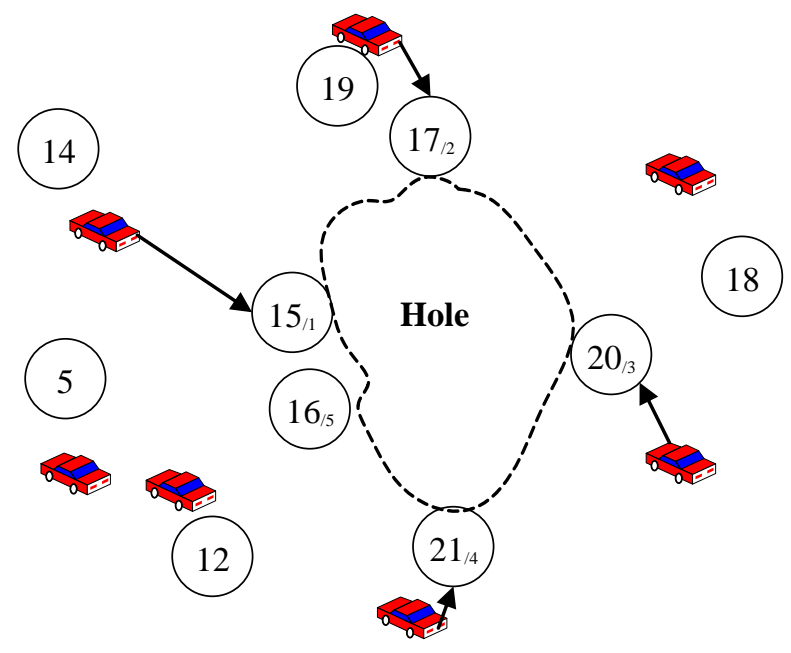

Figure 2. The Group $S$ is Consist of Boundary Nodes 15, 17, 20, 21 and 16

With the presence of obstacles, we also define two sensor connectivity states. Here, the wireless radio of sensor is assumed as a disk model with radius $R_{c}$. If the distance between node $s_{i}$ and $s_{j}$ is smaller than $R_{c}$ and the line segment between $s_{i}$ and $s_{j}$ does not intersect any obstacle, it means $s_{i}$ and $s_{j}$ is connecting. Otherwise, they are disconnected. Notice that, if two sensors are disconnected, it means an obstacle appears between them. In other words, the mobile sensors moving among them may be interrupted by the obstacle. On the basis of the scattered select notion to find an obstacle-free path for mobile sensor. When a mobile sensor is selected moving to the hole, firstly the mobile sensor has to carry out the Dynamic Source Routing protocol (DSR) [12], which can obtain the shortest path between the selected mobile sensor and the hole. The results, except the lower energy consumption, can improve the redundancy mobile sensors distribution in the field uniformly. The advantage of DSR protocol can record the routed path, which can obtain all nodes ID during discovery shortest path. Finally, selected mobile sensors can move to the hole along the shortest path.

$$
A=m \times R_{c}{ }^{2} \times\left(\mathrm{cm}(0), \text { where } \theta=\frac{\pi \times(\pi:-2)}{2 \pi !}\right.
$$




$$
\mathrm{u}=\left|\frac{m \times \cos \pi}{\pi}\right|
$$

The area size of hole is coarse estimation. It assumes a regular polygon is constructed from the $m$ 's boundary nodes and each edge length is $2 R c$, hole's area size will be compute in Eqs. (1); where $m$ is the number of boundary nodes in $S$. The $n$ is calculated in Eqs. (2), which indicate the number of mobile sensors for healing task, the $[\mathrm{x}]$ operation is on the integral function of $x$. We evenly select $n$ out of $m$ boundary nodes to invite the near mobile sensors to move to hole. The flowchart of the boundary nodes dispersion selecting scheme is discussed in Figure 3. The hole healing scheme that we proposed, mobile sensors have shorter movement distance to move to the hole, which because the path is decided by the DSR protocol. In other words, the energy consumption of the mobile sensor is lower and the residual mobile sensors can be uniformly distributed in the surveillance area.

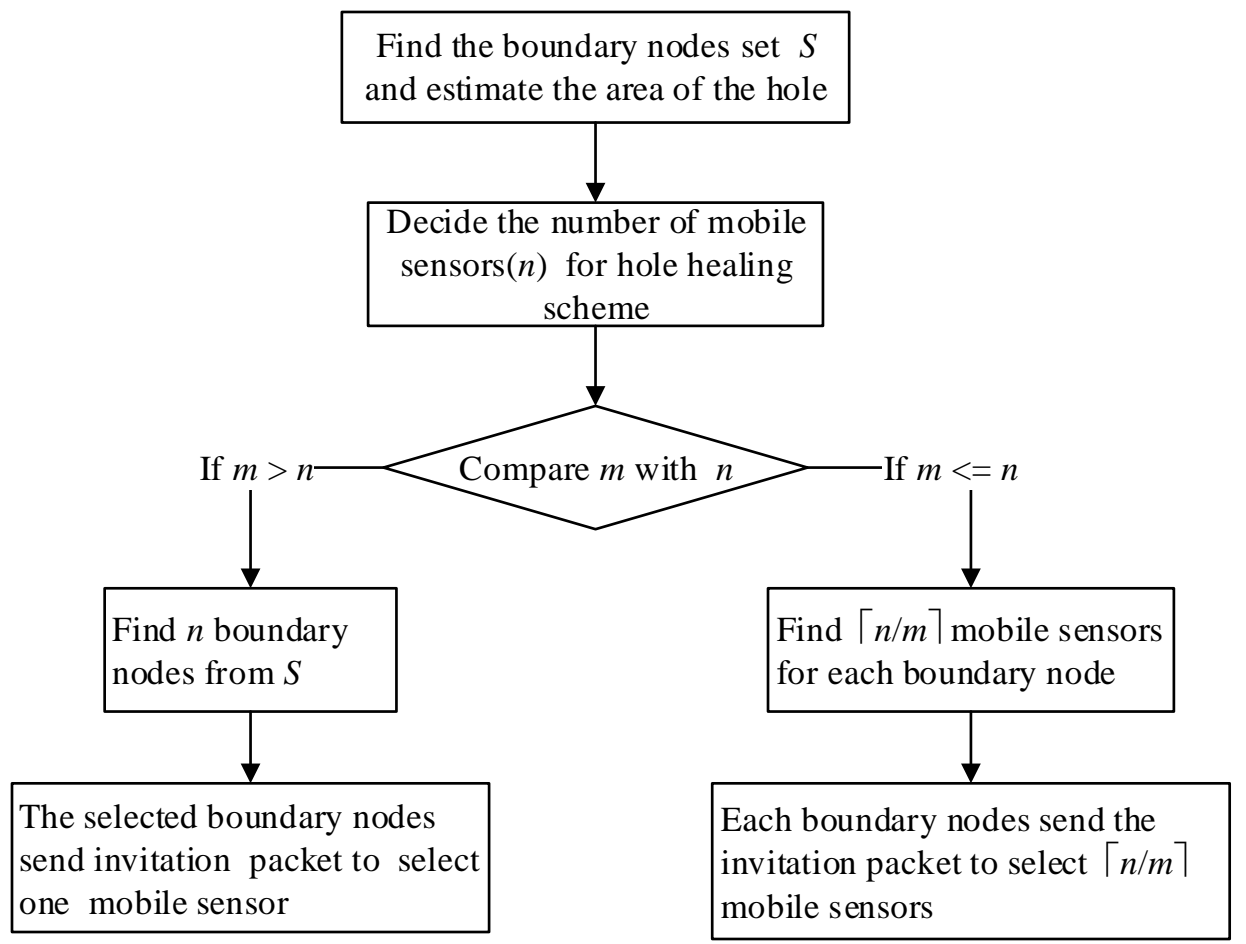

Figure 3. Flowchart of the Participation Boundary Nodes Selected Scheme

\section{Performance Evaluation}

The performance of the proposed OGDD is evaluated and compared with that of the efficient placement and dispatch of sensors (EPDS) [6] and direct direction dispatch scheme (DDDS). The directly direction dispatch scheme is the traditional dispatch method, whose moving is direct direction. A monitored area was simulated, in which sensors are consisted of both static and mobile sensors, 400 and 50 respectively. These sensors were randomly deployed in a $100 \times 100 \mathrm{~m}$ square field with a few obstacles, and the distance between two adjacent sensors is equal to the communication range $\left(R_{c}\right)$ of a sensor. The sink node is located at the lower left corner, partial mobile sensors are allocated close to the sink node and others are uniformly scattered in the square field. The location of the hole is assumed at the upper right corner. For simplicity, the hop counts can be used to represent the mobile sensor 
moved distance, and the unit of energy consumption is assumed as a hop, which expresses unit energy depletion for a mobile sensor moved one-hop distance. These experiments are evaluated 50 times, and the average values are taken.

In the first experiment, results are shown in Figure 4. The dispatch performance about the mobile sensor movement is assessed. The arrival ratio is defined as the ratio of successful move to the destination with total number of move. Our proposed OGDD method and EPDS can achieve the $100 \%$ arrival rate, when the mobile sensors are used. The EPDS is the optimal dispatch scheme to be proposed in [6], which can let the mobile sensor to avoid the obstacles in advancing. However, our proposed mobile sensor routing path is determined by the DSR protocol, therefore the mobile sensors can find an obstacle-free and the shortest path to move to the hole, and the proposed OGDD dispatch scheme is non-centralized, which indicates that the selected mobile sensors are evenly dispersed surrounding the hole.

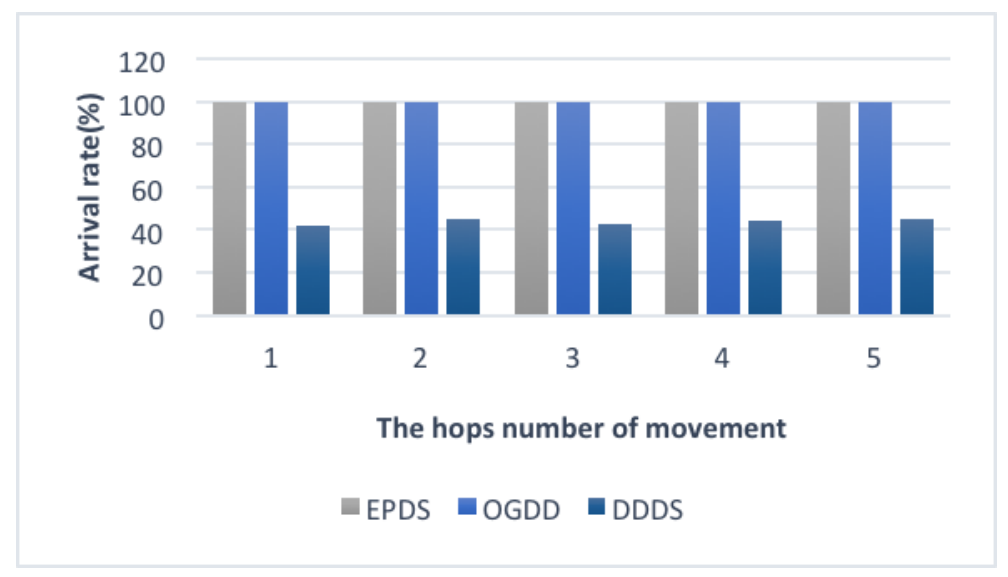

Figure 4. Compare the Relationship of Average Arrival Rate in Three Schemes

Here we will compare the OGDD with EPDS in energy consumption, assumption the area of the hole is constant but the distance is varied between the hole and the sink. Figure 5 shows the distributed dispatch scheme simulation results, which illustrates the results relationship between the position of hole and the average movement distance of the mobile sensors for the holes healing task. Obviously, we proposed the OGDD method in energy consumption is lower than the EPDS method, if the position of hole occurred at the center of the field, that is to say, the position of hole is not adjacent with the field border. The EPDS scheme using to dispatch mobile sensors are mostly coming from between the sink and the hole, which result in the mobile sensors dispatched excessively centralized. On the contrary, in OGDD scheme the assigned mobile sensors are allocated surrounding the hole evenly and the dispatched mobile sensors have to carry out the DSR protocol before moving. Therefore, the dispatching of OGDD is more dispersed and the energy consumption is lower which owing to the mobile sensors shorter movement distance. But when the position of hole is adjacent with the field border or closely to the sink, the OGDD will consume more energy than EPDS scheme because the mobile sensors in OGDD have to move longer path along with the border of hole to the assigned boundary nodes. For example, when the distance between the sink and the hole is 2 hops or 17 hops away, in Figure 5, it showed that the average energy consumption of OGDD scheme wastes larger than the EPDS scheme, because the positions of the holes concerned with two cases are too close to the field border. In addition, regarding the EPDS, owing to the radio disseminate characteristic and the notification packet send by the sink, 
which results in the mobile sensors compete with each other, and there are more interference when the distance of hole is far away from the sink.

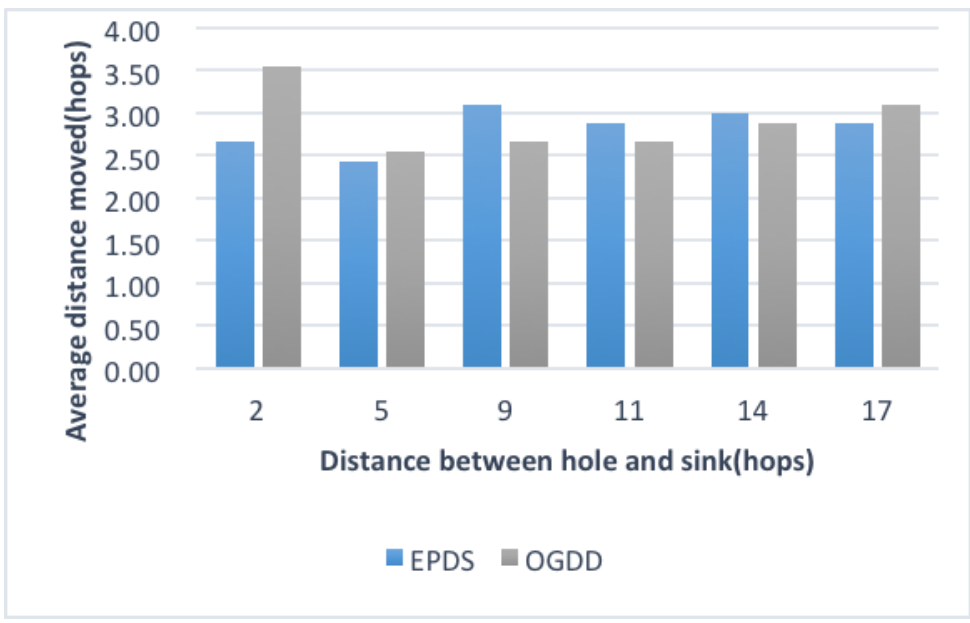

Figure 5. Compare Two Healing Schemes in Energy Consumption on

\section{Conclusion}

The rapid rescue the coverage hole on hybrid sensor network is importance problem in WSN applications. Mobile sensors are advantageous because sensors can dynamically move to cover the uncovered area to increase the coverage. Thus, researchers have been studying the mobile sensor dispatch problem for holes healing task. Generally, optimal schemes need more computing capability of sensors and the geographic information of obstacle. However, sensors usually have resource constraints because the wireless sensors are powered by battery. Therefore the energy, computing capability and communication capability of sensor nodes are limited. In practice, the optimal schemes are hard to implement. Hence, this work designs an obstacle-free and graphic-free decentralized dispatch mobile sensors healing scheme, which is based on the shortest path routing DSR protocol with the objective of low computational complexity and obstacle-free movement for mobile sensors navigation. Simulation results further indicate that the proposed healing scheme can reduce both the average moving distance of mobile sensors and the energy consumption, when the coverage hole occurred in the middle area of the field. But if the coverage hole occurred closely the field border, results show the performance worse than EPDS scheme. Further, we will consider more energy consumption factors, such as nodes computation, data transceivers and interference avoided to adjust the participation sensors for healing task to improve the efficiency.

\section{Acknowledgements}

The authors would like to thank the National Science Council of the Republic of China, Taiwan, for financially supporting this research under Contract No. NCS 102-2219-E-006001. 


\section{References}

[1] D. Wang, B. Xie, and D. P. Agrawal. Coverage and Lifetime Optimization of Wireless Sensor Networks with Gaussian Distribution. IEEE Transactions on Mobile Computing, Vol. 7, No. 12, pp. 1444-1458, December (2008).

[2] C.-F. Huang and Y.-C. Tseng. The Coverage Problem in a Wireless Sensor Network. ACM Mobile Networks and Applications (MONET), special issue on Wireless Sensor Networks, Vol. 10, No. 4 , pp. 519-528, Aug. (2005).

[3] T.-W. Sung and C.-S. Yang. A Cell-based Sensor Deployment Strategy with Improved Coverage for Mobility-Assisted Hybrid Wireless Sensor Networks. International Journal of Ad Hoc and Ubiquitous Computing, Vol. 5, No.3, pp. 189-198. (2010).

[4] F.-T. Lin, T.-W. Sung, C.-Y. Lee and C.-S. Yang. A Delivery Time-aware Hole Healing Scheme for Wireless Sensor Networks. The Fifth International Conference on Ubiquitous Future Networks, (2013) July 2-5; Da Nang, Vietnam.

[5] T.-W. Sung and C.-S. Yang. Voronoi-based Coverage Improvement Approach for Wireless Directional Sensor Networks. Journal of Network and Computer Applications, DOI:10.1016/j.jnca.2013.07.003. (2013).

[6] R.-S. Chang and S.-H. Wang. Self-Deployment by Density Control in Sensor Networks. IEEE Transactions on Vehicular Technology, Vol. 57, No. 3, May (2008).

[7] Y.-C. Wang, C.-C. Hu, and Y.-C. Tseng. Efficient Placement and Dispatch of Sensors in a Wireless Sensor Network. IEEE Transactions on Mobile Computing, Vol. 7, No. 2, Feb. (2008).

[8] J. Kanno, J. G. Buchart, R. R. Selmic, and V. Phoha. Detecting Coverage Holes in Wireless Sensor Networks. 17th Mediterranean Conference on Control \& Automation, (2009) June 24 - 26; Makedonia Palace, Thessaloniki, Greece.

[9] K.Y. Hsieh, Jang-Ping Sheu. Hole Detection and Boundary Recognition in Wireless Sensor Networks. IEEE 20th International Symposium on Personal, Indoor and Mobile Radio Communications, (2009) September 13-16; Tokyo, Japan.

[10] Y. Wang, J. Gao, Joseph S.B. Mitchell. Boundary recognition in sensor networks by topological methods. MobiCom'06, (2006) September 23-26; Los Angeles, California, USA.

[11] N. Heo and P.K. Varshney. Energy-Efficient Deployment of Intelligent Mobile Sensor Networks. IEEE Trans. Systems, Man and Cybernetics_-Part A: Systems and Humans, vol. 35, no. 1, pp. 7892, (2005).

[12] Y. Zou and K. Chakrabarty. Sensor Deployment and Target Localization Based on Virtual Forces. Proc. IEEE INFOCOM, pp. 1293-1303, (2003).

[13] D. B. Johnson and D. A. Maltz, Editor, Mobile Computing, Springer US Publishers, (1996). 
International Journal of Future Generation Communication and Networking Vol.6, No.6 (2013) 